\title{
The Research of Agricultural Expert System based on IOT FuBing ${ }^{1,2}$
}

\author{
1.College of Arts and Science, Yangtze University, Jingzhou434020, China; \\ 2.College of Computer Science, Yangtze University, Jingzhou434020, China. \\ fubing@yangtzeu.edu.cn
}

\begin{abstract}
Keywords:agricultural, expertsystem, mobile device,Internet of Things.
\end{abstract}
\begin{abstract}
Agricultural expert system has been widely used in developed countries, but these cutting-edge technologies have not been universal used in the China's agricultural production, because terminal equipment is inconvenient to carry and complicated to operate for farmers. Under this situation, this thesis explores how to design an agricultural expert system based on IOT, focus on the terminal equipment is easily taken and operated by growers, like mobile devices. The information gathered by mobile devices includes two parts: first, manual input messages, such as taking photos, text message or recording about disease of crops, and second, sensors collect basically plant messages transfer to phone via Bluetooth, such as temperature, humidity, light intensity and the content of CO2.The information would be sent to the database, by the expert system reasoning, or expert personally answering from remote, Growers receive the guidance of scientific production and pest control methods. The research could promote the application of agricultural expert system and improve yields and quality of crops aim at increasing incomes of growers.
\end{abstract}

\section{Introduction}

At present, there are a number of important research results in the field of agricultural informatization around the world, which lay the foundation for the application of information technology based on expert system of the Internet of Things[1] [2].However, in the actual survey, it is found that these sophisticated cutting-edge technologies have not been universal and applied in the agricultural production. The main reason is that computer, network and related knowledge have not enough been universal in China's rural areas. Moreover, experts' diagnostic systems of these agricultural have highly profession and require a higher level of technology for users. We pay particular emphasis on being easy to use on the expert system.Android smart phones with photographs, recordings and other functions are cheap, easy to carry and a wide range using. Also their hardware configurations are high and functions are closer to the PC.Android smartphone is used as the terminal equipment in agricultural expert system, greatly improving its portability and popularity it can combine on-site treatment with remote processing by network and benefit the application and promotion of agricultural expert systems.

Our research combines the Internet of Things, the latest technology of expert systems with convenience to farmers' mobile service. The information gathered by Android-phone includes two parts: first, manual input messages, such as taking photos, text message or recording about disease of crops, and second, sensors collect basically plant messages transfer to phone via Bluetooth, such as temperature, humidity, light intensity and the content of CO2.The information would be sent to the back-end database, by the expert system reasoning, or diagnose of neural network algorithm, or expert personally answering from remote, Growers receive the guidance of scientific production and pest control methods.In our design, farmers first use the analysis of computer expert system or the inference of pest diagnosis based on neural networks about crop growth and pest control problems. Experts of fruit and vegetable would remotely and personally answer the question that cannot be solved or resolved undesirable by expert system of computer.

Design features of mobile platform based on the IOT is easy to be accepted by farmers. For specific circumstances, the process of using is able to accurately manage process of crop cultivation, 
and improve crop yields to achieve production increasing. Researches of the platform have some practical value. It can promote agricultural informatization and accelerate the application and popularization of agricultural expert system.

\section{System design}

\section{A. System structue}

The expert system based Internet of things integrates the wireless sensor network, 4G network and the Internet to realize the farmland air and soil environment data collection and analysis. The system is divided into three layers in our design: the farmland environment information collection layer, the network transport layer and the system application layer. There are mobile devices as nodes to collect sensors ' data, scene photographs and the description voices in the environmental information acquisition layer. In the network transport layer, the environment data collected in the first layer is transmitted to the cloud platform with accessing Internet by 4G wireless communication network. The data would be stored in the database for calculating and analyzing in XML format. Considering the security and the stability of data, the data processing server, the database server and the application server are designed dividedly. The three servers could not only maintain independently in function but also interact for the data and controller. The structure of the system is shown in Fig .1.

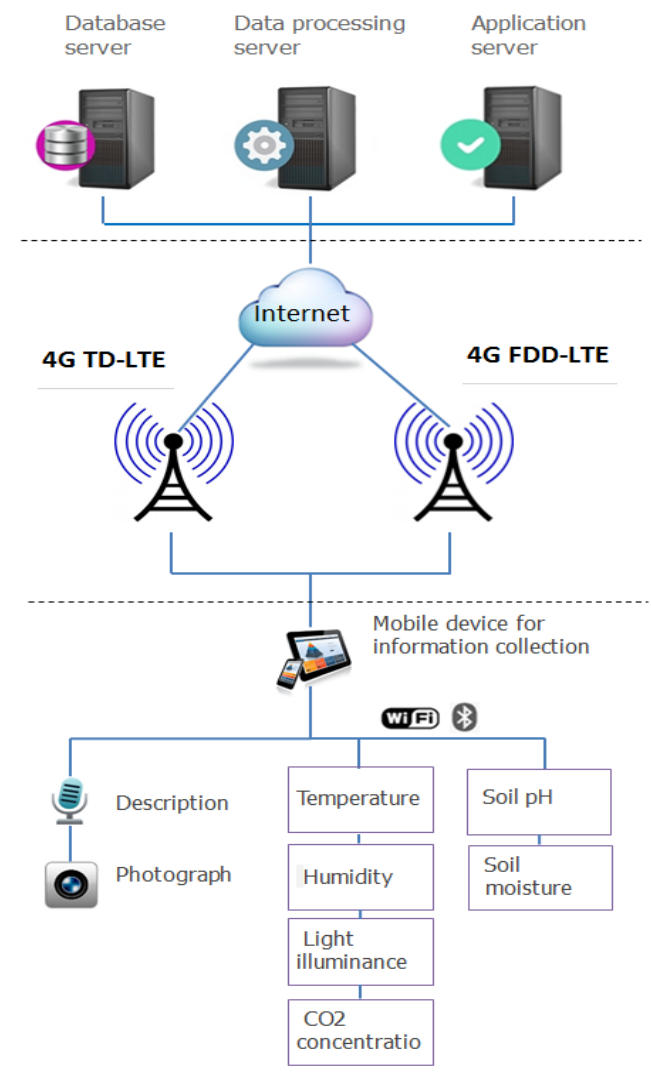

Fig. 1 Systemstructure

\section{B. Function design}

There are 3 parts in the System mainly,includes browsing, gathering and reasoning.

The browsingincludes the overview of botanical features, the farm crop disease to harm andthe insect pest. It is used for expert system to provide crop cultivation include the overview of crop physiology, common pests overview, fertilizer management, water management and other data, it also provides general knowledge for the growth of crops, thereby helping users to enhance further understanding of the crop, and also to solve the general problems of farmers through real-time query data, Improving the utilization efficiency. 
The gathering includes two parts, from human input and sensors respectively, includes the information of temperature, humidity, light intensity, the content of $\mathrm{CO} 2$, crop symptoms, growth stage, etc.

The reasoning has function of disease diagnosisand cultivation process design, includes disease to harm and the insect pest at root, stem, leaf, fruit, etc.

\section{Expert systemdesign}

\section{A. Knowledge base design}

Expert system for fruit- tree trainingis a new research and application field of agriculture expert system [3] [4].According to the known types of fruit diseases, pest species, follow isrepresentation ofknowledge.SymptomMatrix is establishedfor various conditions, and the knowledge of the symptoms is expressedasequation(1).

$$
D_{i}=\left\{a_{1}, a_{2}, a_{3}, \cdots, a_{n}\right\}
$$

$D_{i}$ represents some kind of disease or pests, $a_{1}$ represents a variety of symptoms of $D_{i}$. The weight of all kinds of symptoms is equation (2).

$\mathrm{W}_{\mathrm{i}}=\left\{\mathrm{w}_{1}, \mathrm{w}_{2}, \mathrm{w}_{3}, \cdots, \mathrm{w}_{\mathrm{n}}\right\}$

Experts make Scoreson Symptoms of the disease in eachdisease. The score set is equation(3)

$$
\mathrm{Dc}_{\mathrm{i}}=\left\{\mathrm{c}_{1}, \mathrm{c}_{2}, \mathrm{c}_{3}, \cdots, \mathrm{c}_{\mathrm{n}}\right\}
$$

Diagnostic result set is equation (4).

Result $=(\mathrm{R}, \mathrm{S}, \mathrm{I}, \mathrm{T})$

Symptoms that user imputed,are made to reasoningand do numerical diagnosis with theeachdisease cases in knowledge base. Diagnostic successfully returns diagnostic results.

Rrepresentscredits of $\mathrm{D}_{\mathrm{i}}$ that user' inputs of the diagnosed symptoms; Srepresentsscoresof $\mathrm{D}_{\mathrm{i}}$ that user inputs diagnosed symptom; I represents the description ofthe symptoms ofD $_{\mathrm{i}}$, including the main symptoms, the time of morbidityand so on.T represents treatmentprogram.

\section{B. diagnosis algorithm}

Typically, the search strategiesof case knowledge are mainly nearest neighbor strategy, inductive reasoning strategies, knowledge guiding strategy and template retrieval strategies.Thenearest method is used as the index strategy, by comparing the user's input and knowledge base of the caseto choose the nearest, most similar cases.Numerical diagnostic method is the application of knowledge engineering,making the diagnosis of the disease symptoms to diagnosis of the value. In the diagnosis,diagnostic algorithms with simple, rapid, accurate diagnosis of high are used to make out the diagnostic results in a fuzzy recognition. Diagnostic algorithm is as follows:

According farmer's help information,iretrieve contents for the corresponding disease in the database, to get test cases vectors, asequation (5).

$$
\mathrm{S}_{\mathrm{x}}=\left\{\mathrm{x}_{1}, \mathrm{x}_{2}, \mathrm{x}_{3}, \cdots, \mathrm{x}_{\mathrm{n}}\right\}, \mathrm{x}_{\mathrm{i}}=0 \text { or } 1
$$

As follows, $\mathrm{q}$ is thesimilarity with test cases and case of Knowledge base, for similarity measure we take the nearest neighbor algorithm, namely distance.

$$
\mathrm{q}=1-\sqrt{\sum_{\mathrm{k}=1}^{\mathrm{m}} \mathrm{W}_{\mathrm{k}}^{2} \sum_{\mathrm{k}=1}^{\mathrm{m}} \mathrm{N}_{\mathrm{k}}^{2}}
$$

In (6), $\mathrm{W}_{\mathrm{k}}$ is the weight of the k.th symptoms, $\mathrm{W}_{\mathrm{k}}=\mathrm{P}\left(\mathrm{S}_{\mathrm{k}}\right) / \sum_{\mathrm{x}=1}^{\mathrm{n}} \mathrm{P}\left(\mathrm{S}_{\mathrm{k}}\right), \mathrm{N}_{\mathrm{k}}$ is the similarity between tested cases and cases in knowledge base. If the two symptoms are same, then the value of $\mathrm{N}_{\mathrm{k}}$ is 1 , otherwise $\mathrm{N}_{\mathrm{k}}$ is 0 .

The highest similarity case $\mathrm{D}$ is screenedby calculating with the test case, calculating the credibility of thecase $\rho$, as equation(7).

$$
\rho=\sum_{\mathrm{j}=1}^{\mathrm{n}} \mathrm{Sc}_{\mathrm{j}} / \sum_{\mathrm{j}=1}^{\mathrm{n}} \mathrm{Dc}_{\mathrm{j}}
$$

In (12), $\mathrm{Sc}_{\mathrm{j}}$ is the value that thesymptomNo. $\mathrm{j}$ scores share ratio incases $\mathrm{D}_{\mathrm{j}}$ in test case. $\mathrm{Dc} \mathrm{c}_{\mathrm{j}}$ is the value thatthesymptomNo. $\mathrm{j}$ scores share ratio incases $\mathrm{D}_{\mathrm{j}}$ in knowledge base. 
Finally,to judge whether $\rho$ is bigger thanthreshold0.9,the treatment and diagnosis results would be is given successful.

\section{Conclusion}

Our research was still in the stage of split testing phase, and the system was not yet fully integrated. Moreover, the research involves many technical categories, the integration of 4G, Wi-Fi, Bluetooth, IOT, sensor technology. At present, it is the risk factors to promote a comprehensive project that there are some security threats[5]. And the current cost of operating the new technology is high to be used widely.

However, with the extensive application of the IOT, the cost will be substantially reduced, and its application will be extended to all aspects of agricultural production.

In our research, some agricultural problems have be solved through our system. Also, fruiter experts would answer the problems personally through the remote system based on the IOT.

In the subsequent research, we will not only improve and integrate system, but also increase the online promotion capabilities in fruit and vegetable products.The platform achieves the network services by $4 \mathrm{G}$, and improves the ability of remote service and dealing the problem in real time. The terminal of the system is smart phone, which is easy to carry, treat on-site it is benefit to popularize and promote agricultural expert system.

\section{Acknowledgment}

This research has been supported by the science and technology develop project in Jingzhou.

\section{References}

[1] RomeoJ.,PajaresG.,MontalvoM.,GuerreroJ.M., GuijarroM. and De La Cruz J.M., ”A new Expert System for greenness identification in agricultural images", Expert Systems with Applications, Vol.40, No.6, pp. 2275-2286, May 2013.

[2] KumarVikas, DaveVishal, NagraniRohan, ChaudharySanjay and BhiseMinal,” Crop cultivation information system on mobile devices”,c2013 IEEE Global Humanitarian Technology Conference: South Asia Satellite, GHTC-SAS 2013, pp.196-202, 2013.

[3] LiXinxing, Zhang Lingxian, Fu Zetian and Wen Haojie, "An agricultural knowledge acquisition method based on the coupling of Call center and expert system", Transactions of the Chinese Society of Agricultural Engineering,2011, Vol.27,No.5,pp.174-177.

[4] HouJiajia, LiDongmei,HaoShudong, LiNa and MoQin,” Construction and application of the expert system of diagnosis for orchard pests and diseases”, Journal of Chemical and Pharmaceutical Research, 2013, Vol.5, No.11, pp. 112-117

[5] Yang Guang, Geng Guining, Du Jing, Liu Zhaohui, Han He,"Security threats and measures for theInternet of Things”,Journal of Tsinghua University(Science and Technology)2011, Vol.51, No.10,pp. 1335-1340. 